\title{
Puente adhesivo temporal como solución estética en paciente con fisura de labio y paladar.
}

\section{Temporary adhesive bridge as aesthetic solution in patient withcleft lip palate}

\author{
Noemí Leiva $^{1 *}$, Loreto Saavedra ${ }^{2}$, Francisca Carranza ${ }^{2}$, María Sat ${ }^{2}$ \\ 1. Unidad de Malformaciones Maxilofaciales, \\ Facultad de Odontología, Universidad de Chile Chile \\ 2. Estadía en Unidad de Malformaciones \\ Maxilofaciales 2016-2017, Facultad de \\ Odontología, Universidad de Chile, Chile \\ * Correspondencia Autor: Noemí Leiva | General \\ Holley 2381, oficina 606. Providencia. Santiago \\ de Chile | Teléfono: +56990736159 | e-mail: \\ leivanoemi@yahoo.com \\ Trabajo recibido el 26/06/2017. \\ Aprobado para su publicación el 28/01/2018

\begin{abstract}
RESUMEN
Las dificultades por ausencia de una pieza dentaria anterior en un paciente con fisura de labio y paladar operado y en período de crecimiento requieren una solución estética inmediata posterior al retiro de aparatología fija de ortodoncia y en espera de una solución definitiva, sin embargo, ante escenarios como éste son útiles materiales de restauración que cumplan con indicaciones como rapidez en el trabajo, de tipo adhesivos reforzados con fibras de polietileno, altamente estéticos, manipulables y útiles en moldeabilidad.

Se presenta un caso donde se utilizó resina compuesta, una cinta reforzada de fibra de polietileno, para la realización de un puente de reposición temporal de dos piezas dentarias anteriores en un paciente con diagnóstico de fisura de labio y paladar unilateral como una solución muy estética, no invasiva, provisional en espera del término del crecimiento para una rehabilitación definitiva.
\end{abstract} \\ PALABRAS CLAVE \\ Fisura labiopalatina; Unión Adhesiva; Estética Dental.
}

Rev. Clin. Periodoncia Implantol. Rehabil. Oral Vol. 11(2); 106-108, 2018.

\begin{abstract}
There are several therapeutic alternatives for the rehabilitation of absent permanentteeth, but these alternatives are limited when the piece has an important esthetic component and must be performed in developing patients. Such is the case of patientswith cleft palate, for whom it is essential to use a highly esthetic material and torehabilitate edentulous spaces, usually an incisor, once the orthodontic treatment iscompleted and as a temporary solution awaiting the definitive rehabilitation. In these situations, restorative materials reinforced with polyethylene fibers are useful.In this case, a reinforced tape of polyethylene fiber was used as a temporary replacement bridge of two anterior teeth in a patient with a diagnosis of cleft lip and unilateral palate as an esthetic, non-invasive and temporary solution until his growth and a definitive rehabilitation.
\end{abstract}

KEY WORDS

Cleft lip palate; Resin bonded; Dental esthetic.

Rev. Clin. Periodoncia Implantol. Rehabil. Oral Vol. 11(2); 106-108, 2018.

\section{INTRODUCCIÓN}

Los motivos de la ausencia de dientes pueden ser congénitas (agenesias), caries o traumatismos. De éstas, la ausencia congénita por fisura labio palatina es común ${ }^{(1)}$. En la mayoría de los pacientes jóvenes con diagnóstico de labio leporino y/o paladar con fisura operado, la rehabilitación del espacio desdentado en el sector anterior es una gran necesidad. No sólo debe cumplir con las condiciones de estética y funcionalidad, sino que además debe ser flexible y acompañar el movimiento de los segmentos óseos cuando aún no se ha realizado el injerto óseo alveolar ${ }^{(2)}$. El tratamiento en estos casos debe ser lo más conservador posible, evitando intervenir las piezas adyacentes a la zona desdentada, más aún cuando están sanas. Por tal motivo, la técnica adhesiva utilizando un sistema de fibras constituye una alternativa viable de rehabilitación provisoria durante el periodo de crecimiento y en espera de la rehabilitación definitiva ${ }^{(2,3)}$.

Los composites reforzados con fibras (CRF) son materiales en base a resina que contienen fibras que mejoran sus propiedades físicas ${ }^{(3)}$. Se han añadido a estas resinas diferentes tipos de fibras tales como fibras de vidrio, carbono y de polietileno. Estas últimas han mostrado una gran ventaja en comparación a las anteriormente desarrolladas, principalmente debido a las propiedades en relación a la resistencia al impacto, el módulo de elasticidad y la resistencia a la flexión que le confieren a las resinas compuestas. Constituye un material biocompatible, estético, translúcido, prácticamente incoloro y no se visualiza dentro del composite o $\operatorname{acrílico}^{3,}$, 4). Por medio de los procedimientos estándar, recomendados por cada fabricante según la marca comercial que se esté usando, la resina o composite puede adherirse a: esmalte, dentina, metal, porcelana, composite y resinas acrílicas. Ribbond ${ }^{\circledR}$, un ejemplo de estas fibras reforzadas con polietileno, se adapta fácilmente a la morfología del diente y al contorno del arco dental, ya que no posee memoria ${ }^{(4,5,6)}$. Ensayos clínicos han utilizado este tipo de fibra de polietileno en su presentación comercial Ribbond $^{\circledR}$, y su aplicación ha sido descrita en diversas áreas de la Odontología(4,5,7) (fig. 1).

Cuando los pacientes portadores de fisura de labio y velopalatina se encuentran entre los 12 y 17 años de edad, es indispensable realizar 

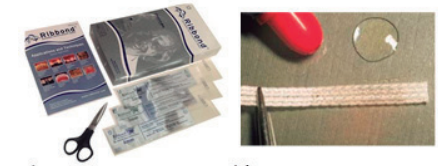

b)

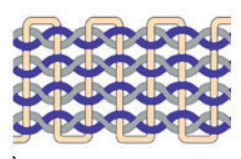

c)

Figura 1. a) Presentación comercial b) Imagen de Cinta reforzada con polietileno c) Esquema de estructura interna: tejido de fibras de polietileno enlazables y refor-zadas de ultra alta resistencia.

una rehabilitación provisoria que permita mejorar la estética y otorgar una solución transitoria y confortable para, una vez finalizado el crecimiento, completar el tratamiento odontológico(2). Este requisito es fundamental cuando el tratamiento ortodóncico está en su fase final y se ingresa en un periodo de espera para la rehabilitación definitiva, ya sea mediante una prótesis parcial fija plural o implante óseo integrado.

En la gran mayoría de estos pacientes jóvenes encontraremos que las piezas adyacentes a la zona desdentada se encuentran sanas. Por lo tanto, un tratamiento conservador en este caso sería el uso de la técnica adhesiva mediante fibras de polietileno cubiertas de plasma en conjunto con resina compuesta; y de esta forma poder confeccionar un puente adhesivo temporal, utilizando el composite como material para tallar el diente que va adherido a la cinta.

\section{CASO CLÍNICO}

El paciente I.G. con diagnóstico de fisura de labio y paladar unilateral izquierdo, fue tratado por un equipo multidisciplinario por su anomalía. Ingresó a los 7 años de edad a la especialidad de Ortodoncia. Tras un tratamiento de ortodoncia interceptiva y posteriormente ortodoncia correctiva con aparatología fija, multibracketts, es derivado a los 16 y 17 años de edad a Implantología para evaluar la posibilidad de reposición mediante implante óseo integrado de pieza 2.2 ausente. Los especialistas determinaron que aún no es posible realizar rehabilitación mediante implantes y se debe esperar 12 a 18 meses (fig. 2).

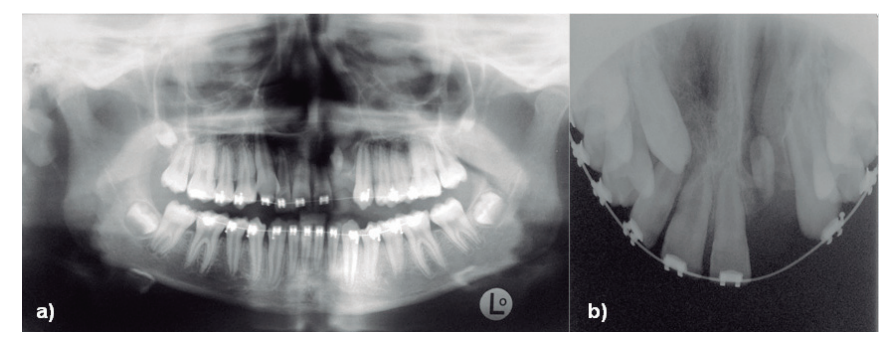

Figura 2. a) Radiografía Panorámica a los 11 años que confirma ausencia de pie-za 2.2. b) Radiografía Oclusal Superior a los 15 años de edad. Control de injerto óseo alveolar en la fisura labio palatina. Se aprecia estabilización de segmentos maxilares, continuidad del arco. Se observa área de menor densidad proyectada en zona de la fisura izquierda y ausencia de pieza 2.2.

Ante la imposibilidad de realizar un tratamiento definitivo en esos momentos, se decidió rehabilitar temporalmente el vano desdentado mediante un puente adhesivo de dos piezas dentarias. Presentaba una correcta higiene oral, ausencia de enfermedad sistémica, ausencia de enfermedad periodontal, dientes vitales adyacentes a la fisura, sin caries ni restauraciones, ausencia de onicofagia. Es importante mencionar que una prótesis fija plural convencional no es lo indicado en este caso, debido a la edad del paciente, el crecimiento y la maduración de los tejidos pulpares y periodontales que no han finalizado.

Inicialmente se llevó a cabo un registro fotográfico del paciente, profilaxis de ambas arcadas y se realizó la construcción del puente de acuerdo a los siguientes pasos ${ }^{(2)}$ :

1. Aislación relativa del campo de trabajo intraoral.

2. Los dientes fueron limpiados y secados.

3. El esmalte fue grabado usando ácido ortofosfórico al $37 \%$ por 30 segundos, luego se lavó por 30 segundos y se secó con aire, según las indicaciones del material

4. Se aplicó adhesivo universal, se secó con aire y fotopolimerizó por 20 segundos, siguiendo las indicaciones del fabricante.

5. Se distribuyó una capa de resina compuesta en el canal virtual donde se colocará la cinta y se seleccionó el grosor $3 \mathrm{~mm}$. Éste fue humectado con el líquido adhesivo e inmediatamente ubicado sobre el composite no polimerizado, uniendo ambos dientes con el trozo de la cinta previamente medida con un trozo de papel aluminio y cortado con la tijera de serrucho ${ }^{\circledR}$. La resina compuesta fue polimerizada junto con la cinta y el adhesivo. Se fue agregando resina compuesta tallando directamente dos dientes debido a la extensión del vano desdentado y se aplicó la cinta vestibular de refuerzo eligiendo la de $2 \mathrm{~mm}$ de grosor (fig. 3).

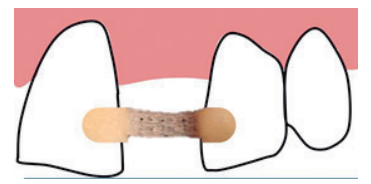

Figura 3. Dibujo que grafica el posicionamiento de la cinta reforzada con polietileno por palatino.

6. Terminación, completando el fotocurado.

7. Se retiró la aislación relativa y se adaptó la zona gingival con resina compuesta para terminar el póntico. Se procedió a pulir y se comprobó que la cinta no presentara zonas expuestas al medio bucal (figs. 4-6).

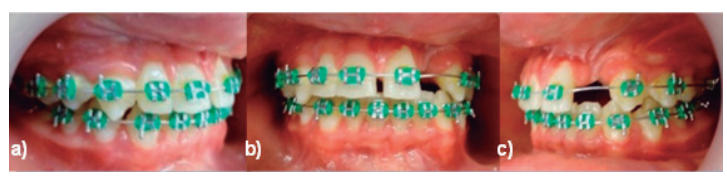

Figura 4. Fotografías intraorales previas a instalación de póntico. a) Vista lateral derecha; b) Vista frontal; c) Vista lateral izquierda. Se observa zona de la fisura con ausencia de pieza 2.2

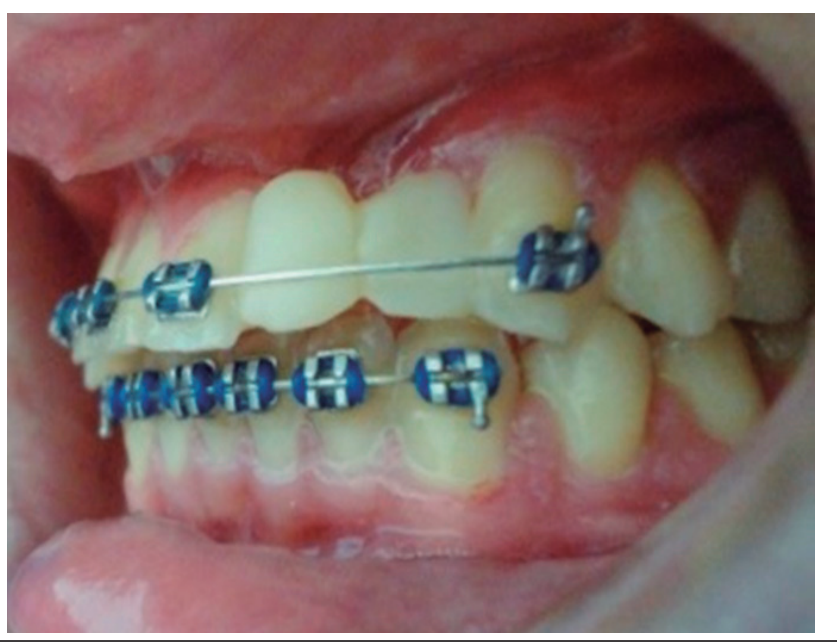

Figura 5. Vista lateral izquierda de puente de reposición temporal. Se mantuvo el segmento de aparato fijo por un mes.

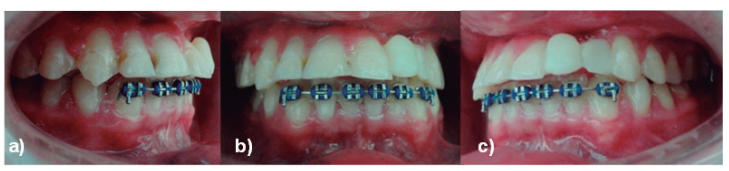

Figura 6. Fotografías intraorales posteriores a rehabilitación temporal mediante póntico. a) Vista lateral derecha. b) Vista frontal. c) Vista lateral izquierda. Se observa puente pulido y terminado.

\section{DISCUSIÓN}

Para los pacientes en crecimiento y desarrollo, esta alternativa terapéutica podría considerarse como un tratamiento provisional que proporcionaría una función y estética aceptables mediante la sustitución de los dientes y tejidos faltantes hasta que se pueda realizar una restauración definitiva. Para llevarlo a cabo, puede utilizarse un diente de acrílico o resina compuesta(6,8).

En este caso, la aplicación de la cinta reforzada con fibra de polietileno en adición con resina ha permitido la reposición temporal de dos piezas dentarias, mediante la confección de un puente adhesivo altamente estético y con adecuadas propiedades mecánicas en un paciente con diagnóstico de fisura de labio y paladar operado y con tratamiento ortodóncico terminado. Este tratamiento proporciona bajos costos de tratamiento; en caso de fractura de la resina del diente o desgaste, el 
aparato puede repararse fácilmente. No hay necesidad de eliminar tejido dentario, haciendo que la técnica sea reversible y conservadora. También satisface las expectativas estéticas de los pacientes, siendo cómodo y no cubriendo el paladar como la haría una placa removible.

Un estudio clínico retrospectivo llevado a cabo por Piovesan y cols ${ }^{(9)}$ sugirieron que una prótesis parcial fija confeccionada con composite reforzados con fibras de polietileno podría ser una alternativa funcional y estética para reemplazar un diente perdido, no dificulta la posición de la lengua al hablar, ya que mantiene la línea del arco rehabilitado. Unlu y Belli ${ }^{(10)} \|$ legaron a la conclusión de que este tipo de prótesis reforzadas con polietileno funcionaron adecuadamente durante un periodo de seguimiento clínico de 3 años. En otro informe, se describe una tasa de supervivencia funcional de $95 \%$ después de un período de seguimiento de 4.3 años $^{(7)}$. La longevidad de estas prótesis parciales reforzadas con fibras depende de variados factores, incluyendo el material, el arco rehabilitado, factores propios del paciente y de la confección. Strassler y cols. ${ }^{(11)}$ reportaron una vida útil de estas restauraciones fijas reforzadas con fibras de 5 a 10 años. Entre los factores del paciente se pueden mencionar la higiene oral, hábitos de alimentación, medidas preventivas, fluoración, cumplimiento en los controles mensuales por los primeros seis meses para posteriormente ingresar al tratamiento definitivo, cooperación durante el tratamiento y condiciones orales. Todos estos son temas relevantes al momento de considerar la durabilidad ${ }^{(2)}$.

La última etapa del tratamiento en estos pacientes consiste en la rehabilitación oral de la zona de la fisura con agenesia de la pieza dentaria anterior mediante implantes o prótesis fija. Debido al alto impacto estético y funcional, adquiere relevancia para ellos el poder realizar este tipo de rehabilitación provisoria, ya que si comparamos con una prótesis removible, ésta ocupa espacio en el paladar y dificulta la fonación, es incómoda, y sólo deberán esperar el término de su crecimiento para concluir este proceso, así evitar estar este período con ausencia de diente en esa zona, lo que altera la estética, se pierde la línea del arco dentario, altera la sonrisa influyendo en la interacción con sus pares, quienes a esa edad ya tienen dentición completa definitiva.

\section{CONFLICTO DE INTERESES}

Los autores declaran no tener ningún conflicto de intereses.

\section{Bibliografía}

1. Gupta A, Yelluri RK, Munshi A. Fiber-reinforced composite resin bridge: A treatment option in children. Int J Clin Pediatr Dent. 2015;8(1):62-65.

2. Leiva N, Corsini R, Nieto E. Puente adhesivo en paciente con labio leporino y fisura velopalatina: Una solución temporal con compromiso estético. Rev Clin Periodoncia Implantol Rehabil Oral. 2013;6( 1 ):33-37.

3. Belli S, Eskitascioglu G. Biomechanical properties and clinical use of a polyethylene fibre post-core material. Int Dentistry SA. 2006;8 (3):20-26.

4. Maden $\mathrm{E}$, Altun $\mathrm{C}$. Use of polyethylene fiber (Ribbond) in pediatric dentistry. Arch Clin Exp Surg. 2012;1(2):110-115

5. Garoushi S, Lassila L, Vallittu PK. Resin-bonded fiber-reinforced composite for direct replacement of missing anterior teeth: A clinical report. Int J Dent. 2011;2011:845420

6. Chaudhary V, Shrivastava B, Bhatia HP, Aggarwal A, Singh AK, Gupta N Multifunctional Ribbond. A versatile tool. J Clin Pediatr Dent. 2012; 36(4):325-328.
7. Tuloglu N, Bayrak S, Tunc ES. Different clinical applications of bondable reinforcement Ribbond in pediatric dentistry. Eur J Dent. 2009; 3: 329-334.

8. Khetarpal A, Talwar S, Verma M. Creating a single-visit, fibre-reinforced composite resin bridge by using a natural tooth pontic: A viable alternative to a PFM bridge. J Clin Diagn Res. 2013:7(4):772-775.

9. Piovesan E, Demarco F, Piva E. Fiber-reinforced fixed partial dentures: A preliminary restrospective clinical study. J Appl Oral Sci, 2006;14(2):100-104.

10. Unlu N, Belli S. Three-year clinical evaluation of fiber-reinforced composite fixed partial dentures using prefabricated pontics. J Adhes Dent. 2006;8(3):183-188.

11. Strassler HE, Dzakovich JJ. High-strength fiber-reinforced fixed partial dentures with a wear-resistant composite resin. Compend Contin Educ Dent. 2005;26(10):716 718-22, 724. 